\title{
Measuring Intrinsic Volumes in Digital 3d Images
}

\author{
Katja Schladitz ${ }^{1}$, Joachim Ohser ${ }^{2}$, and Werner Nagel ${ }^{3}$ \\ ${ }^{1}$ Fraunhofer-Institut für Techno- und Wirtschaftsmathematik \\ Fraunhofer-Platz 1 \\ 67663 Kaiserslautern, Germany \\ katja.schladitz@itwm.fraunhofer.de \\ ${ }^{2}$ Fachhochschule Darmstadt \\ Schöfferstraße 3 \\ 64295 Darmstadt, Germany \\ ohser@fh-darmstadt.de \\ ${ }^{3}$ Friedrich-Schiller-Universität \\ Fakultät für Mathematik und Informatik \\ 07740 Jena, Germany \\ nagel@minet.uni-jena.de
}

\begin{abstract}
The intrinsic volumes - in 3d up to constants volume, surface area, integral of mean curvature, and Euler number - are a very useful set of geometric characteristics. Combining integral and digital geometry we develop a method for efficient simultanous calculation of the intrinsic volumes of sets observed in binary images. In order to achieve consistency in the derived intrinsic volumes for both foreground and background, suitable pairs of discrete connectivities have to be used. To make this rigorous, the concepts discretization w.r.t. an adjacency system and complementarity of adjacency systems are introduced.
\end{abstract}

\section{Introduction}

With the fast development of new materials like foams or fiber reinforced composites there is a growing need for non-destructive testing and structure characterization. In particular, computer tomography is now able to produce high quality $3 \mathrm{~d}$ images of very fine structures, yielding the demand for subsequent quantitative analysis.

In many applications, geometric characteristics of the whole structure have to be measured from the given image. A very attractive set of geometric characteristics are the intrinsic volumes (or quermassintegrals or Minkowski functionals). In $3 \mathrm{~d}$, they are, up to constants, volume, surface area, integral of mean curvature, and Euler number. For fibrous structures, the integral of mean curvature yields the total fiber length without need to segment individual fibers.

The Crofton formulae boil down computing the intrinsic volumes to computing Euler numbers in lower dimensional intersections. Discretization of these formulae (see Section 4) combined with an efficient calculation of the Euler numbers in the intersections yield a fast algorithm for simultaneously determining 
the intrinsic volumes based on observations in a digital image. The backbone of the Euler number calculation are thorough investigations of digital connectivity and consistency from [1,2,3, summarized in Section 3,

The consistency results for the Euler number of foreground and background established in 2] carry over to all intrinsic volumes. A remarkable observation in this context is that the 18-neighborhood can not be used for consistent estimation of the intrinsic volumes. This counteracts the fact that $(18,6)$ is considered to be a 'good pair' in digital topology, see e.g. [4, Chapter 7].

In $3 \mathrm{~d}$, the presented method's algorithmic core consists in a convolution of the binary image with a $2 \times 2 \times 2$ mask, resulting in an 8 bit gray value image [5]. All further steps are based solely on the gray value histogram whose size does not depend on image size or content. Thus the advantage over other methods for computing the intrinsic volumes 6,7, are simplicity and speed of the algorithm. The surface area is measured directly from the binary volume image without need to approximate the surface. Similar methods are [8] and 9], see Section 6] for a comparison.

\section{Section Lattices and Translative Complements}

In this paper, we restrict ourselves to the three-dimensional cubic primitive lattice $\mathbb{L}^{3}=a \mathbb{Z}^{3}, a>0$, where $\mathbb{Z}$ is the set of integers. For a more general approach see 10. Let $u_{1}, u_{2}, u_{3}$ denote the standard unit vector basis of the threedimensional Euclidean space $\mathbb{R}^{3}$. The closed unit cell of $\mathbb{L}^{3}$ is the Minkowski sum $C=\left[0, a u_{1}\right]+\left[0, a u_{2}\right]+\left[0, a u_{3}\right]$. Its volume is vol $C=a^{3}$. We denote by $\mathcal{F}^{0}$ the set of vertices of a polyhedron, in particular $\mathcal{F}^{0}(C)=\{0, a\}^{3}$. The set of all lattice cells covers $\mathbb{R}^{3}=\bigcup_{x \in \mathbb{L}^{3}}(C+x)$.

The Crofton formulae for computing the intrinsic volumes of a set $X \subset \mathbb{R}^{3}$ use section profiles of $X$ on affine subspaces of $\mathbb{R}^{3}$. In order to obtain a digitized version, we introduce section lattices of $\mathbb{L}^{3}$ and their translative complements in analogy to linear subspaces and their orthogonal complements:

Definition 1. A pair $\left(\mathbb{L}^{k}{ }^{T} \mathbb{L}^{3-k}\right), k=1,2$, is called a $k$-dimensional section lattice $\mathbb{L}^{k}$ equipped with the translative complement ${ }^{T} \mathbb{L}^{3-k}$, if there exists a basis $v_{1}, v_{2}, v_{3}$ of $\mathbb{L}^{3}$ with

(i) $\mathbb{L}^{k}=\left(v_{1}, v_{k}\right) \mathbb{Z}^{k}$

(ii) ${ }^{T} \mathbb{L}^{3-k}=\left(v_{k+1}, v_{3}\right) \mathbb{Z}^{3-k}$,

(iii) there is an $x \in \mathcal{F}^{0}(\check{C})$ with $\left\{v_{1}, v_{k}\right\} \subset \mathcal{F}^{0}(C+x)$ where $\check{C}$ is the reflection of $C$ at the origin, $\breve{C}=-C$.

Condition (iii) ensures that integration over 'local knowledge' on the image data is possible as needed later. The translative complement ${ }^{T} \mathbb{L}^{3-k}$ has properties similar to those of the orthogonal complement of a linear subspace:

1. $\mathbb{L}^{k} \cap \cap^{T} \mathbb{L}^{3-k}=0$,

2. If $x_{1}, x_{2} \in{ }^{T} \mathbb{L}^{3-k}, x_{1} \neq x_{2}$, then $\left(\mathbb{L}^{k}+x_{1}\right) \cap\left(\mathbb{L}^{k}+x_{2}\right)=\emptyset$,

3. $\mathbb{L}^{3}=\bigcup_{x \in \mathbb{T}^{3-k}}\left(\mathbb{L}^{k}+x\right)$. 
However, the translative complement is not necessarily uniquely determined. Nevertheless, choosing one of the translative complements arbitrarily turns out to work for all considerations presented in the following. For $\mathbb{L}^{3}$, there are $13 \mathrm{sec}-$ tion lattices $\mathbb{L}_{i}^{k}$ for both $k=1$ and $k=2$. This restriction is due to condition (iii).

Table 1. The bases of the 13 section lattices $\mathbb{L}_{i}^{k}$ of $\mathbb{L}^{3}=\mathbb{Z}^{3}$ and a possible translative complement ${ }^{T} \mathbb{L}_{i}^{k}$ for $k=1$ (left), and $k=2$ (right)

\begin{tabular}{r|c|c}
$i$ & basis of $\mathbb{L}_{i}^{1}$ & basis of ${ }^{T} \mathbb{L}_{i}^{2}$ \\
\hline 1 & $\left\{u_{1}\right\}$ & $\left\{u_{2}, u_{3}\right\}$ \\
2 & $\left\{u_{2}\right\}$ & $\left\{u_{1}, u_{3}\right\}$ \\
3 & $\left\{u_{3}\right\}$ & $\left\{u_{1}, u_{2}\right\}$ \\
4 & $\left\{u_{1}+u_{2}\right\}$ & $\left\{u_{1}, u_{3}\right\}$ \\
5 & $\left\{-u_{1}+u_{2}\right\}$ & $\left\{u_{1}, u_{3}\right\}$ \\
6 & $\left\{u_{1}+u_{3}\right\}$ & $\left\{u_{1}, u_{2}\right\}$ \\
7 & $\left\{-u_{1}+u_{3}\right\}$ & $\left\{u_{1}, u_{2}\right\}$ \\
8 & $\left\{u_{2}+u_{3}\right\}$ & $\left\{u_{1}, u_{3}\right\}$ \\
9 & $\left\{-u_{2}+u_{3}\right\}$ & $\left\{u_{1}, u_{3}\right\}$ \\
10 & $\left\{-u_{1}+u_{2}+u_{3}\right\}$ & $\left\{u_{1}, u_{2}\right\}$ \\
11 & $\left\{-u_{1}+u_{2}+u_{3}\right\}$ & $\left\{u_{1}, u_{2}\right\}$ \\
12 & $\left\{u_{1}-u_{2}+u_{3}\right\}$ & $\left\{u_{1}, u_{2}\right\}$ \\
13 & $\left\{u_{1}+u_{2}-u_{3}\right\}$ & $\left\{u_{1}, u_{2}\right\}$
\end{tabular}

\begin{tabular}{r|c|c}
$i$ & basis of $\mathbb{L}_{i}^{2}$ & basis of ${ }^{T} \mathbb{L}_{i}^{1}$ \\
\hline 1 & $\left\{u_{1}, u_{2}\right\}$ & $\left\{u_{3}\right\}$ \\
2 & $\left\{u_{1}, u_{3}\right\}$ & $\left\{u_{2}\right\}$ \\
3 & $\left\{u_{2}, u_{3}\right\}$ & $\left\{u_{1}\right\}$ \\
4 & $\left\{u_{1}, u_{2}+u_{3}\right\}$ & $\left\{u_{3}\right\}$ \\
5 & $\left\{u_{1},-u_{2}+u_{3}\right\}$ & $\left\{u_{3}\right\}$ \\
6 & $\left\{u_{2}, u_{1}+u_{3}\right\}$ & $\left\{u_{3}\right\}$ \\
7 & $\left\{u_{2},-u_{1}+u_{3}\right\}$ & $\left\{u_{3}\right\}$ \\
8 & $\left\{u_{3}, u_{1}+u_{2}\right\}$ & $\left\{u_{1}\right\}$ \\
9 & $\left\{u_{3},-u_{1}+u_{2}\right\}$ & $\left\{u_{1}\right\}$ \\
10 & $\left\{u_{1}+u_{3}, u_{2}+u_{3}\right\}$ & $\left\{u_{3}\right\}$ \\
11 & $\left\{-u_{1}+u_{3}, u_{2}+u_{3}\right\}$ & $\left\{u_{3}\right\}$ \\
12 & $\left\{-u_{1}+u_{3},-u_{2}+u_{3}\right\}$ & $\left\{u_{3}\right\}$ \\
13 & $\left\{u_{1}+u_{3},-u_{2}+u_{3}\right\}$ & $\left\{u_{3}\right\}$
\end{tabular}

\section{Adjacency and Euler Number}

The Crofton formulae reduce the measurement of the intrinsic volumes $V_{3-k}$ of a poly-convex set $X$ to measuring the Euler number $\chi$ of section profiles of $X$ in $k$-dimensional sections, $k=1,2$. Thus it is essential to know how the Euler number $\chi(X \cap(L+y))$ can be measured when instead of $X \cap(L+y)$ only the observation $X \cap\left(\mathbb{L}^{k}+y\right)$ on a translated section lattice is available with $L=\operatorname{span} \mathbb{L}^{k}$ and $y \in{ }^{T} \mathbb{L}^{3-k}$.

The problem of measuring the Euler number based on images was considered by several authors [11, 12, 6, 7]. Here we apply the concept of adjacency systems from [1,2,3]. Good pairs of adjacencies for foreground and background allow consistent calculation of the Euler number, see 3.3. It turns out that this condition differs from the usually demanded Jordan surface theorem as the 18neighborhood can not be used for consistent calculation of the Euler number. Note that for dimensions three and higher, a complete description of good adjacencies is not yet known.

\subsection{Discretization with Respect to an Adjacency System}

Let $\mathbb{L}^{k}$ be a (section) lattice with the basis $v_{1}, v_{k}$ and the unit cell $C^{k}$. The vertices $x_{j}=\sum_{i=1}^{k} \lambda_{i} v_{i}$ of $C^{k}$ are indexed with $j=\sum_{i=1}^{k} 2^{i-1} \lambda_{i}, \lambda_{i} \in\{0,1\}$. Clearly, the unit cell $C^{k}$ has $2^{k}$ vertices $x_{j} \in \mathcal{F}^{0}\left(C^{k}\right), j=0, \ldots, 2^{k}-1$. Analogously, we introduce the index of a subset $\xi \subseteq \mathcal{F}^{0}\left(C^{k}\right)$. Let $\mathbf{1}$ denote the indicator 
function of a set, i. e. $\mathbf{1}(x \in \xi)=1$ if $x \in \xi$ and $\mathbf{1}(x \in \xi)=0$ otherwise. An index $\ell$ is assigned to $\xi$, and we write $\xi_{\ell}$ if $\ell=\sum_{j=0}^{2^{k}-1} 2^{j} \mathbf{1}\left(x_{j} \in \xi\right)$, i. e. $\ell \in\{0, \ldots, \nu\}$ with $\nu=2^{2^{k}}-1$. Note that $\xi_{0}=\emptyset, \xi_{\nu}=\mathcal{F}^{0}\left(C^{k}\right)$, and $\xi_{\nu-\ell}=\xi_{\nu} \backslash \xi_{\ell}$. The $\xi_{\ell}$ can be considered as a local pixel configuration of the foreground of a $k$-dimensional binary image. Finally, we introduce the convex hulls $F_{\ell}=\operatorname{conv} \xi_{\ell}$ forming convex polytopes with $F_{\ell} \subseteq C^{k}$ and $\mathcal{F}^{0}\left(F_{\ell}\right) \subseteq \mathcal{F}^{0}\left(C^{k}\right), \ell=1, \ldots, \nu$. Let $\mathcal{F}^{j}(F)$ denote the set of all $j$-dimensional faces of a convex polytope $F$. For a set $\mathbb{F}$ of convex polytopes write $\mathcal{F}^{j}(\mathbb{F})=\cup\left\{\mathcal{F}^{j}(F): F \in \mathbb{F}\right\}$ for the set of all $j$-faces. Now we are able to equip the lattice $\mathbb{L}^{k}$ with a (homogeneous) adjacency system defining the neighborhood of the lattice points.

Definition 2. Let $\mathbb{F}_{0} \subseteq\left\{F_{0}, \ldots, F_{\nu}\right\}$ be a set of convex polytopes $F_{\ell}=$ conv $\xi_{\ell}$ with the properties

(i) $\emptyset \in \mathbb{F}_{0}, C \in \mathbb{F}_{0}$,

(ii) if $F \in \mathbb{F}_{0}$ then $\mathcal{F}^{i}(F) \subset \mathbb{F}_{0}$ for $i=0, \ldots, \operatorname{dim} F$,

(iii) if $F_{i}, F_{j} \in \mathbb{F}_{0}$ and $F_{i} \cup F_{j}$ is convex then $F_{i} \cup F_{j} \in \mathbb{F}_{0}$.

Then the system $\mathbb{F}_{0}$ is a local adjacency system and $\mathbb{F}=\bigcup_{x \in \mathbb{L}^{k}} \mathbb{F}_{0}+x$ is called an adjacency system of the lattice $\mathbb{L}^{k}$.

From condition (i) it follows immediately that $\mathcal{F}^{0}(\mathbb{F})=\mathbb{L}^{k}$. The pair $\Gamma=$ $\left(\mathcal{F}^{0}(\mathbb{F}), \mathcal{F}^{1}(\mathbb{F})\right)$ consisting of the set $\mathcal{F}^{0}(\mathbb{F})$ of nodes and the set $\mathcal{F}^{1}(\mathbb{F})$ of edges is said to be the neighborhood graph of $\mathbb{F}$. Due to homogeneity $(\Gamma+x=\Gamma$, $x \in \mathbb{L}^{k}$ ), all nodes have the same valence - the connectivity of $\mathbb{L}^{k}$. Note that for $n>2$ there can be two or more adjacency systems having the same neighborhood graph. In other words, an adjacency system $\mathbb{F}$ is not uniquely determined by $\Gamma$.

Examples of Adjacency Systems for $k=3$ :

The 6-adjacency is generated from the unit cell $C^{3}, \mathbb{F}_{0}=\cup_{j=0}^{3} \mathcal{F}^{j}\left(C^{3}\right)$.

The 14.1-adjacency is generated from the tessellation of $C^{3}$ into the 6 tetrahedra $F_{139}, F_{141}, F_{163}, F_{177}, F_{197}, F_{209}$ which are the convex hulls of the configurations

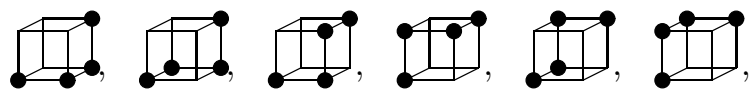

i. e. $\mathbb{F}_{0}$ consists of all $j$-faces of the tetrahedra, $j=0, \ldots, 3$, and their convex unions. The edges of the corresponding neighborhood graph $\Gamma$ are the edges of $C^{3}$, the face diagonals of $C^{3}$ containing the origin 0 , the space diagonal of $C^{3}$ containing 0 , and all their lattice translations. The degree of $\Gamma$ is 14 .

The 14.2-adjacency is generated from the tetrahedra $F_{43}, F_{141}, F_{147}, F_{169}$, $F_{177}$, and $F_{212}$ which are the convex hulls of
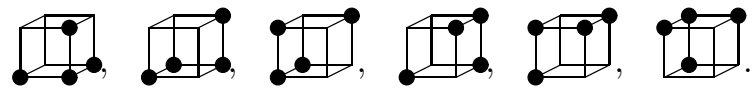

The corresponding neighborhood graph $\Gamma$ differs from that one for 14.1 in the choice of one face diagonal of $C^{3}$ not containing 0 . 
The 26-adjacency is given by $\mathbb{F}_{0}=\left\{F_{0}, \ldots, F_{255}\right\}$.

Definition 3. The discretization $X \sqcap \mathbb{F}$ of a compact set $X \subset \mathbb{R}^{3}$ with respect to a given adjacency system $\mathbb{F}$ is defined as the union of all $j$-faces of the elements of $\mathbb{F}$ for which all the vertices hit $X$, i. e.

$$
X \sqcap \mathbb{F}=\cup\left\{F \in \mathbb{F}: \mathcal{F}^{0}(F) \subseteq X\right\} .
$$

This means that a 'brick' $F \in \mathbb{F}$ is a subset of the discretization of $X$ if and only if all vertices of $F$ belong to $X$. The discretization is obtained from the observation of the set on the lattice, i.e. $X \sqcap \mathbb{F}=\left(X \cap \mathbb{L}^{3}\right) \sqcap \mathbb{F}$.

\subsection{Euler Number}

Since $X \sqcap \mathbb{F}$ forms a (not necessarily convex) polyhedron on span $\mathbb{L}^{k}$, the number of elements of $\mathcal{F}^{j}(X \sqcap \mathbb{F})$ is finite. Therefore, the Euler number $\chi(X \sqcap \mathbb{F})$ can be computed via the Euler-Poincaré formula,

$$
\chi(X \sqcap \mathbb{F})=\sum_{j=0}^{k}(-1)^{j} \# \mathcal{F}^{j}(X \sqcap \mathbb{F}) .
$$

In order to apply a 'local method' for measuring the intrinsic volumes we deduce now a local version. The discretization of a local configuration $\xi_{\ell}=X \cap C^{k} \cap \mathbb{L}^{k}$ of $X \cap \mathbb{L}^{k}$ is $\xi_{\ell} \sqcap \mathbb{F}=(X \sqcap \mathbb{F}) \cap C^{k}=X \sqcap \mathbb{F}_{0}$. We compute weights for the edge correction using $\kappa F=\min \left\{j:\right.$ there is a $G \in \mathcal{F}^{j}\left(C^{k}\right)$ with $\left.F \subseteq G\right\}$. Now define the edge-corrected localization $\chi_{0}$ of $\chi$ as

$$
\chi_{0}\left(\xi_{\ell} \sqcap \mathbb{F}\right):=\sum_{j=0}^{k}(-1)^{j} \sum_{F \in \mathcal{F}^{j}\left(\xi_{\ell} \sqcap \mathbb{F}\right)} 2^{\kappa F-k}, \quad \ell=0, \ldots, \nu .
$$

Then, additivity and translation invariance of the Euler number and the fact that $X \sqcap \mathbb{F}=\left(X \cap \mathbb{L}^{k}\right) \sqcap \mathbb{F}$ yield

$$
\begin{aligned}
\chi(X \sqcap \mathbb{F}) & =\sum_{x \in \mathbb{L}^{k}} \chi_{0}\left(C^{k} \cap((X \sqcap \mathbb{F})-x)\right) \\
& =\sum_{x \in \mathbb{L}^{k}} \sum_{\ell=0}^{\nu} \chi_{0}\left(\xi_{\ell} \sqcap \mathbb{F}\right) \mathbf{1}\left(\xi_{\ell}+x \subseteq X\right) \mathbf{1}\left(\xi_{\nu-\ell}+x \subseteq X^{c}\right) \\
& =\sum_{\ell=0}^{\nu} \chi_{0}\left(\xi_{\ell} \sqcap \mathbb{F}\right) \underbrace{\sum_{x \in \mathbb{L}^{k}} \mathbf{1}\left(\xi_{\ell}+x \subseteq X\right) \mathbf{1}\left(\xi_{\nu-\ell}+x \subseteq X^{c}\right)}_{=: h_{\ell}} .
\end{aligned}
$$

Thus the Euler number can be written as a scalar product, $\chi(X \sqcap \mathbb{F})=w h$, where the components $w_{\ell}=\chi_{0}\left(\xi_{\ell} \sqcap \mathbb{F}\right)$ of the vector $w=\left(w_{\ell}\right)$ depend on $\mathbb{F}$, but they are independent of $X$. On the other hand, the vector $h=\left(h_{\ell}\right)$ is independent of $\mathbb{F}$; its components $h_{\ell}$ can be computed very efficiently from 'local information' about $X \cap \mathbb{L}^{3}$. 


\subsection{Complementarity}

It is well-known that choosing an adjacency system $\mathbb{F}$ for the discretization of $X$ implies an $\mathbb{F}_{c}$ for the discretization of the complementary set $X^{c}$. In other words, if the 'foreground' $X \cap \mathbb{L}^{k}$ is connected with respect to $\mathbb{F}$ then the 'background' $\overline{X^{c}} \cap \mathbb{L}^{k}$ must be connected with respect to a complementary adjacency $\mathbb{F}_{c}$. The usual criterion for complementarity is the Jordan surface theorem (JordanBrouwer theorem), see e.g. [13. However, when aiming at computation of the intrinsic volumes, another criterion seems to be more appropriate: In the continuous case the consistency relation

$$
\chi(X)=(-1)^{k+1} \chi\left(\overline{X^{c}}\right)
$$

holds for all compact, poly-convex and topologically regular sets $X \subset \operatorname{span} \mathbb{L}^{k}$, see [2. In the discrete case this leads to:

Definition 4. The pair $\left(\mathbb{F}, \mathbb{F}_{c}\right)$ is called a pair of complementary adjacency systems if

$$
\chi(X \sqcap \mathbb{F})=(-1)^{k+1} \chi\left(X^{c} \sqcap \mathbb{F}_{c}\right)
$$

for all compact $X \subset \mathbb{R}^{3}$. An adjacency system $\mathbb{F}$ is called self-complementary if $\chi(X \sqcap \mathbb{F})=(-1)^{k+1} \chi\left(X^{c} \sqcap \mathbb{F}\right)$ for all compact $X$.

For a given adjacency system $\mathbb{F}$, existence of a complementary adjacency system $\mathbb{F}_{c}$ is not guaranteed. Even worse, until now, there is no constructive way to find the complementary system $\mathbb{F}_{c}$. However, most known 'good' pairs of adjacencies w.r.t. Jordan curve or surface theorems are complementary in the sense of our definition, too. Complementarity of adjacency systems can be checked the following way: Let $h$ be defined as in (3). Then for $h^{c}=\left(h_{\ell}^{c}\right)$ with $h_{\ell}^{c}=$ $\sum_{x \in \mathbb{L}^{k}} \mathbf{1}\left(\xi_{\ell}+x \subseteq X^{c}\right) \mathbf{1}\left(\xi_{\nu-\ell}+x \subseteq X\right)$ we obtain the relationship $h_{\ell}=h_{\nu-\ell}^{c}$, $\ell=0, \ldots, \nu$. Using (3) and Definition 4 one can easily prove

Lemma 1. Let $\mathbb{F}$ and $\mathbb{F}_{c}$ be two adjacency systems and let $w$ and $w^{c}$ be the vectors with the coefficients $w_{\ell}=\chi_{0}\left(\xi_{\ell} \sqcap \mathbb{F}\right)$ resp. $w_{\ell}^{c}=\chi_{0}\left(\xi_{\ell} \sqcap \mathbb{F}_{c}\right), \ell=0, \ldots, \nu$. Then $\left(\mathbb{F}, \mathbb{F}_{c}\right)$ is a pair of complementary adjacency systems if and only if

$$
w_{\ell}=(-1)^{k+1} w_{\nu-\ell}^{c}, \quad \ell=0, \ldots, \nu .
$$

Examples for 3d: The 6-adjacency is complementary to the 26-adjacency. The 14.1- and the 14.2-adjacencies are constructed to be self-complementary, see 223. However, the 18-adjacency is not complementary to the 6-adjacency although they are 'Jordan-Brouwer-complementary', see e.g. 13. In order to see this, define $\mathbb{F}_{18}$ to be one of the 18-adjacencies with the neighborhood graph $\Gamma^{\prime}=$ $\left(\mathbb{L}^{3}, \mathcal{F}^{1}\right)$ where $\mathcal{F}^{1}$ consists of all edges and face diagonals of the cells of $\mathbb{L}^{3}$. Independent of the choice of $\mathbb{F}_{18}$ we get e.g. $\chi_{0}\left(\mathbb{\square} \sqcap \mathbb{F}_{18}\right)=\frac{1}{4}$ since the space diagonals of the unit cell do not belong to $\mathbb{F}_{18}$. On the other hand we get for the complementary configuration $\chi_{0}\left(\mathbb{R} \cap \mathbb{F}_{6}\right)=-\frac{3}{4}$ where $\mathbb{F}_{6}$ is defined as in Section 3.1 Thus, for the pair $\left(\mathbb{F}_{18}, \mathbb{F}_{6}\right)$, the necessary condition for complementarity (4) is violated. 
Remark: The example $\left(\mathbb{F}_{18}, \mathbb{F}_{6}\right)$ shows that complementarity in the sense of Definition 4 clearly differs from 'Jordan-Brouwer-complementarity'. So far, there is no general result about the relationship of the two conditions known. Jordan surface theorems for the two 14-adjacencies are expected to hold and subject of further research.

\section{Intrinsic Volumes of Poly-Convex Sets}

Consider a poly-convex set $X \subset \mathbb{R}^{3}$. The intrinsic volumes $V_{j}, j=0, \ldots, 3$ are up to constant factors - the volume $V(X)$, the surface area $S(X)=2 V_{2}(X)$, the integral of mean curvature $M(X)=\pi V_{1}(X)$, [14, p. 210] and the Euler number $\chi(X)=V_{0}(X)$. By means of the Crofton formula, the intrinsic volumes can be written as

$$
\frac{1}{2} V_{3-k}(X)=\int_{\mathcal{L}^{k}} \underbrace{\int_{\perp_{L}} \chi(X \cap(L+y)) d \lambda_{\perp_{L}}(y)}_{p_{X}^{k}(L)} d \mu(L), \quad k=1,2,
$$

where $\mathcal{L}^{k}$ is the set of all $k$-dimensional linear subspaces of $\mathbb{R}^{3},{ }^{\perp} L$ denotes the orthogonal complement of $L \in \mathcal{L}^{k}, \lambda_{\perp_{L}}$ is the $3-k$-dimensional Lebesgue measure on ${ }^{\perp} L, \mu$ denotes the rotation invariant probability measure on $\mathcal{L}^{k}, \mu\left(\mathcal{L}^{k}\right)=1$.

Remark: Note that the Crofton formula is also the base of stereological formulae for the intrinsic volumes, see e.g. [15].

Here, the set $X$ is observed in an image (a finite subset of the lattice $\mathbb{L}^{3}$ ) only. This implies that the integrand in the Crofton formulae (5) is known for only a finite number of elements of $\mathcal{L}^{k}$, and the translation $L+y$ is possible for discrete values of $y$, only. That is, both integrals in (5) are approximated by sums. Furthermore, the intersection $(X-y) \cap L$ must be replaced by its discretization $(X-y) \sqcap \mathbb{F}^{k}$ with respect to an adjacency system $\mathbb{F}^{k}$ in $\mathbb{L}^{k}$ where $L=\operatorname{span} \mathbb{L}^{k}$, and the translations $y$ are from ${ }^{T} \mathbb{L}^{3-k}$ instead of ${ }^{\perp} L$, where ${ }^{T} \mathbb{L}^{3-k}$ is a translative complement according to Definition 1 .

\subsection{Discretization of the Translative Integral}

Let $C^{k}$ and ${ }^{T} C^{3-k}$ be the unit cells of $\mathbb{L}^{k}$ and ${ }^{T} \mathbb{L}^{3-k}$, respectively. Denote by proj ${ }^{T} C^{3-k}$ the orthogonal projection of ${ }^{T} C^{3-k}$ onto ${ }^{\perp} L$. Its volume is vol $\operatorname{proj}{ }^{T} C^{3-k}=\operatorname{vol} C / \operatorname{vol} C^{k}$.

Then - in analogy to the rectangular quadrature rule - the inner integral in the Crofton formula (5) can be approximated by

$$
\begin{aligned}
p_{X}^{k}(L) & \left.\left.\approx \frac{\operatorname{vol} C}{\operatorname{vol} C^{k}} \sum_{y \in \mathbb{L}^{3-k}} \chi((X-y) \cap L)\right) \approx \frac{\operatorname{vol} C}{\operatorname{vol} C^{k}} \sum_{y \in \mathbb{L}^{3-k}} \chi\left((X-y) \sqcap \mathbb{F}^{k}\right)\right) \\
& =\frac{\operatorname{vol} C}{\operatorname{vol} C^{k}} \sum_{x \in \mathbb{L}^{3}} \chi_{0}\left(C^{k} \cap\left((X-x) \sqcap \mathbb{F}^{k}\right)\right):=\tilde{p}_{X}^{k}(L)
\end{aligned}
$$


The volume of $\operatorname{proj}^{T} C^{3-k}$ and thus $\tilde{p}_{X}^{k}(L)$ do not depend on the particular choice of ${ }^{T} \mathbb{L}^{3-k}$. From the local Euler-Poincaré-Formula (2) it follows that

$$
\sum_{x \in \mathbb{L}^{3}} \chi_{0}\left(C^{k} \cap\left((X-x) \sqcap \mathbb{F}^{k}\right)\right)=w h
$$

where the vector $w$ corresponds to the adjacency system $\mathbb{F}^{k}$ and $h$ can be computed via

$$
h_{\ell}=\sum_{x \in \mathbb{L}^{3}} \mathbf{1}\left(\xi_{\ell}+x \subset X\right) \mathbf{1}\left(\xi_{\nu-\ell}+x \subset X^{c}\right), \quad \ell=0, \ldots, \nu .
$$

Remark: For an effective algorithmic implementation it is useful to use one vector $\bar{h}$ with (7) which has to be determined just once for given set $X$ and lattice $\mathbb{L}^{3}$. In order to use it for the dimensions $k=1,2$ and the different directions of sections as well as for the computing the Euler number in 3d according to (3), the vectors $\bar{w}$ of weights can be adapted appropriately [5, 10, such that

$$
\sum_{x \in \mathbb{L}^{3}} \chi_{0}\left(C \cap\left((X-x) \sqcap \mathbb{F}^{k}\right)\right)=\bar{w} \bar{h} .
$$

$\bar{h}$ can be interpreted as the gray value histogram of the gray value image obtained by convolution of the binary image with a suitable $2 \times 2 \times 2$ mask.

\subsection{Discretization of the Integral over All Subspaces}

As a consequence of the observation of $X$ on $\mathbb{L}^{3}$ and condition (iii) in Definition 1 an approximation of $p_{X}^{k}$ is known for only finitely many subspaces $L_{i}=\operatorname{span} \mathbb{L}_{i}^{k}, i=1, \ldots, 13$, see Table 1. Hence, an appropriate approximation of $\int_{\mathcal{L}^{k}} p_{X}^{k}(L) d \mu(L)$ is needed. Applying a simple quadrature we get

$$
\int_{\mathcal{L}^{k}} p_{X}^{k}(L) d \mu(L) \approx \int_{\mathcal{L}^{k}} \tilde{p}_{X}^{k}(L) d \mu(L) \approx \sum_{i=1}^{13} \gamma_{i}^{(k)} \tilde{p}_{X}^{k}\left(L_{i}\right)
$$

where $\gamma_{i}^{(k)}$ are the weights corresponding to the applied quadrature rule. The choice of these weights is not trivial since the $L_{1}, \ldots, L_{13}$ are not uniformly scattered in $\mathcal{L}^{k}$ and moreover, the measurement values $\tilde{p}_{X}^{k}\left(L_{i}\right)$ for the $p_{X}^{k}\left(L_{i}\right)$ are not of the same precision for different subspaces.

The weights $\gamma_{i}^{(k)}$ can be chosen as follows, see [5]: For $k=1$ the unit sphere $S^{2}$ is divided into Voronoï cells with respect to the point field $L_{1} \cap S^{2}, \ldots, L_{13} \cap S^{2}$ containing 26 points. Then the weight $\gamma_{i}^{(1)}$ is the sum of the areas (Hausdorff measure) of the two Voronoï cells corresponding to the two points of $L_{i} \cap S^{2}$ divided by the surface area of $S^{2}$. For $k=2$, the same is done with ${ }^{\perp} L_{i}$ instead of $L_{i}$. The numerical values are $\gamma_{i}^{(k)}=0.045778$ for $i=1,2,3 ; \gamma_{i}^{(k)}=0.036981$ for $i=4, \ldots, 9 ; \gamma_{i}^{(k)}=0.035196$ for $i=10, \ldots, 13$. 
Summarizing formulas (5), (6), and (10) we obtain the approximation $\hat{V}_{3-k}(X)$ of $V_{3-k}(X)$ as

$$
\frac{1}{2} \hat{V}_{3-k}(X)=\sum_{i=1}^{13} \gamma_{i}^{(k)} \frac{\operatorname{vol} C}{\operatorname{vol} C_{i}^{k}} \sum_{x \in \mathbb{L}^{3}} \chi_{0}\left(C_{i}^{k} \cap\left((X-x) \sqcap \mathbb{F}_{i}^{k}\right)\right), \quad k=1,2
$$

where $\mathbb{F}_{i}^{k}$ is an adjacency system on the respective section lattice $\mathbb{L}_{i}^{k}$ and $C^{k}$ its unit cell. Using this approximation, consistent measurement of the intrinsic volumes for both foreground and background is possible:

Theorem 1. Let $\left(\mathbb{F}_{i}^{k}, \mathbb{F}_{c, i}^{k}\right)$ be pairs of complementary adjacency systems on the lattices $\mathbb{L}_{i}^{k}, i=1, \ldots, 13$, where $X$ and $X^{c}$ are discretized w.r.t. the $\mathbb{F}_{i}^{k}$ and the $\mathbb{F}_{c, i}^{k}$, respectively. If $X$ is compact then the following consistency relation holds:

$$
\hat{V}_{n-k}(X)=(-1)^{k+1} \hat{V}_{n-k}\left(X^{c}\right), \quad k=1,2 .
$$

This follows directly from Definition 4

From (6), the remark in Section 4.1, (7), (8), and (10) it can be seen that the two intrinsic volumes $V_{1}$ and $V_{2}$, and thus the integral of the mean curvature $M$ and the surface area $S$, can be computed by scalar products, $\hat{S}(X)=v^{(1)} \bar{h}$ and $\hat{M}(X)=v^{(2)} \bar{h}$, respectively, where

$$
v^{(1)}=4 \sum_{i=1}^{13} \frac{\operatorname{vol} C}{\operatorname{vol} C_{i}^{1}} \gamma_{i}^{(1)} \bar{w}_{i}^{(1)}, \quad v^{(2)}=2 \pi \sum_{i=1}^{13} \frac{\operatorname{vol} C}{\operatorname{vol} C_{i}^{2}} \gamma_{i}^{(2)} \bar{w}_{i}^{(2)},
$$

and $\bar{w}_{i}^{(1)}, \bar{w}_{i}^{(2)}$ are the vectors corresponding to $\bar{w}$ (as in (9) for $\mathbb{L}_{i}^{1}$ and $\mathbb{L}_{i}^{2}$, see also [5].

\section{Congruence Classes of Configurations}

In this section, we derive another version of (11) using congruence classes of the local pixel configurations $\xi_{\ell}$ w.r.t. rigid motions and counting $\mathbf{1}\left(\xi_{\ell}+x \subseteq X\right)$ instead of $\mathbf{1}\left(\xi_{\ell}+x \subseteq X\right) \mathbf{1}\left(\xi_{\ell}^{c}+x \subseteq X^{c}\right)$, comparable to [8, 9 .

To this end, chose a pair of complementary adjacency systems $\left(\mathbb{F}, \mathbb{F}_{c}\right)$ for the lattice $\mathbb{L}^{3}$. As in Section 4.2 the section lattices $\mathbb{L}_{i}^{k}$ of $\mathbb{L}^{3}$ are equipped with pairs $\left(\mathbb{F}_{i}^{k}, \mathbb{F}_{c, i}^{k}\right)$ of complementary adjacency systems, $i=1, \ldots, 13, k=1,2$. It is assumed that the section lattices $\mathbb{L}_{i}^{k}$ and the adjacency systems $\mathbb{F}_{i}^{k}, \mathbb{F}_{c, i}^{k}$ are chosen such that for each element $F$ of $\mathbb{F}_{i}^{k} \cup \mathbb{F}_{c, i}^{k}$ there exists a translation $x \in \mathbb{L}^{3}$ such that $F+x \subset C$. (Note that not necessarily $C^{k} \subset C$.) Then it is sufficient to consider local configurations $\xi_{\ell} \subseteq \mathcal{F}^{0}(C), \ell=0, \ldots, 255$.

Consider first replacing $\mathbf{1}\left(\xi_{\ell} \subset X\right) \mathbf{1}\left(\xi_{\ell}^{c} \subset X^{c}\right)$ in (3) and (8)), respectively, by $\mathbf{1}\left(\xi_{\ell} \subset X\right)$. For each set $\xi \subseteq \mathcal{F}^{0}(C), \xi^{c}=\mathcal{F}^{0}(C) \backslash \xi$ and a point $y \in \xi^{c}$ we have

$\mathbf{1}\left(\xi \subset X, \xi^{c} \subset X^{c}\right)=\mathbf{1}\left(\xi \subset X, \xi^{c} \backslash\{y\} \subset X^{c}\right)-\mathbf{1}\left(\xi \cup\{y\} \subset X, \xi^{c} \backslash\{y\} \subset X^{c}\right)$. 
Table 2. The coefficients $g_{3 j}$ of the 21 congruence classes of the local pixel configurations in 3d-images. The columns of $g_{0 j}$ correspond to $\left(\mathbb{F}_{26}, \mathbb{F}_{6}\right),\left(\mathbb{F}_{14.1}, \mathbb{F}_{14.1}\right)$, $\left(\mathbb{F}_{14.2}, \mathbb{F}_{14.2}\right)$, and $\left(\mathbb{F}_{6}, \mathbb{F}_{26}\right)$, in this order. $g_{1 j}=g_{2 j}=0$ for the congruence classes 11-21. Note that the weights are those in (11). That is, the configurations are counted if the black dots are foreground and the other vertices are background.

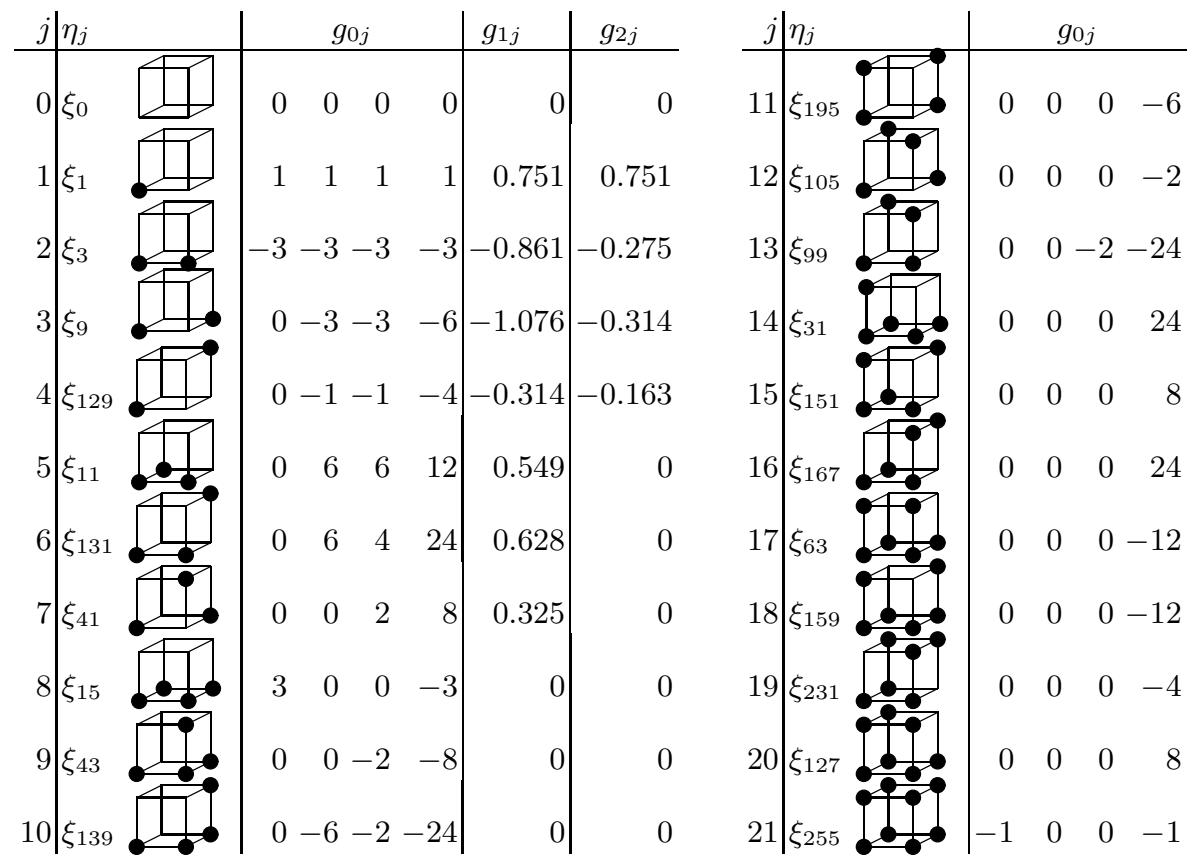

Recursion and translation by $x$ yield

$$
h_{\ell}=\sum_{x \in \mathbb{L}^{3}} \mathbf{1}\left(\xi_{\ell} \subset X-x, \xi_{255-\ell} \subset X-x\right)=\sum_{m=0}^{255} q_{m \ell} \sum_{x \in \mathbb{L}^{3}} \mathbf{1}\left(\xi_{m} \subset X-x\right) \text {, }
$$

$\ell=0, \ldots, 255$, where the $q_{m \ell}$ are integers with $q_{\ell \ell}=1$ for $\ell=0, \ldots, 255$ and $q_{m \ell}=0$ for $m<\ell$. Further, $\sum_{m=0}^{255} q_{m \ell}=0$ for $\ell=0, \ldots, 254$, as follows from the case $X=C$.

Finally, we average the approximations of the intrinsic volumes w.r.t. rotations and inversions that leave the lattice $\mathbb{L}^{3}$ invariant, i.e. w.r.t. the symmetry group $\left\{\theta_{1}, \ldots, \theta_{48}\right\}$ of the octahedron. Let $D_{0}, \ldots, D_{21}$ be the congruence classes of $\left\{\xi_{0}, \ldots, \xi_{255}\right\}$ w.r.t. translations and the octahedral group and let $\left\{\eta_{0}, \ldots, \eta_{21}\right\}$ be a system of representatives, $\eta_{\ell} \in D_{\ell}$. Now, using the coefficients

$$
g_{3-k, j}=\frac{2}{a^{3-k}} \sum_{i=1}^{13} \gamma_{i}^{(k)} \frac{\operatorname{vol} C}{\operatorname{vol} C_{i}^{k}} \sum_{\ell=0}^{255} \bar{q}_{j \ell} \chi_{0}\left(\xi_{\ell} \sqcap \mathbb{F}_{i}^{k}\right), k=1,2,
$$


and

$$
g_{0 j}=\sum_{\ell=0}^{255} \bar{q}_{j \ell} \chi_{0}\left(\xi_{\ell} \sqcap \mathbb{F}\right)
$$

with $\bar{q}_{j \ell}=\sum_{m=0}^{255} q_{m \ell} \mathbf{1}\left(\xi_{m} \in D_{j}\right)$ the approximations of the intrinsic volumes (11) and (3) can be rewritten as

$$
\tilde{V}_{3-k}(X)=a^{3-k} \sum_{j=0}^{21} \frac{1}{48} \sum_{i=1}^{48} \sum_{x \in \mathbb{L}^{3}} \mathbf{1}\left(\theta_{i}^{-1} \eta_{j} \subset X-x\right) g_{3-k, j}, \quad k=1,2,3 .
$$

The $g_{3-k, j}$ are normalized such that they are independent of the lattice distance $a$ and $\tilde{V}_{3-k}(X)$ is the mean of $\hat{V}_{3-k}(X)$ w.r.t. the octahedral group.

For particular cases, the coefficients $g_{3-k, j}$ can easily be computed and be presented in tables. Table 2 contains the $g_{3 j}$ for the pairs $\left(\mathbb{F}, \mathbb{F}_{c}\right)$ of complementary adjacency systems from Section 3.1. The coefficients $g_{1 j}$ and $g_{2 j}$ are computed for the section lattices listed in Table 1. The 2-adjacency is applied for $k=1$ and the 6 -adjacency is applied for $k=2$. The weights $\gamma_{i}^{(k)}$ are chosen as described in 4.2

\section{Discussion}

We introduce a new method for measuring the intrinsic volumes based on weighted local $2 \times 2 \times 2$ configurations. Due to the restriction to these small configurations, an efficient and simple algorithm can be derived. Given a proper choice of connectivities for foreground and background, consistency of the results for foreground and background can be ensured. This is remarkable in particular for the Euler number, as many other algorithms ignore this.

3d imaging techniques like computed tomography and nano-tomography using transmission electron micorscopy combined with focused ion beam sample preparation often produce anisotropic lattices. The results presented in this paper carry over to these cases as well as higher dimensions, see [10]. Note that for anisotropic lattices, the sets of weights $\gamma_{i}^{(k)}$ for $k=1,2$ from 4.2 do not coincide anymore.

There are various methods of surface estimation based on weights for local pixel configurations, see e.g. 8, 9. In Table 3 the weights $b_{j}^{(\mathrm{L})}$ published in [9] are compared with the weights $b_{j}$ computed from the coefficients $g_{2 j}$ given in Table 2. The surface area weights for complementary representatives $\eta_{j}^{c}$ are the same as for $\eta_{j}$.

Clearly, the weights differ, for some of the directions considerably. In 10, multi-grid convergence of the surface area approximation as given by (13) is shown for an important class of random closed sets (Boolean models). A necessary condition for this is $\sum_{j=0}^{21} g_{2 j} b_{j}=0$, obviously violated by the weights $b_{j}^{(\mathrm{L})}$. 
Table 3. Weights for the surface area depending on local configurations: top: the weights from [9], bottom: the weights computed from the coefficients $g_{2 j}$ given in Table 2. Note that the weights here are to be used with (13). That is, the black dots have to be foreground while there is no condition on the others.

\begin{tabular}{|c|c|c|c|c|c|c|c|c|c|c|c|c|c|c|}
\hline$j$ & 0 & 1 & 2 & 3 & 4 & 5 & 6 & 7 & 8 & 9 & 10 & 11 & 12 & 13 \\
\hline$\underline{\eta_{j}}$ & 旡 & 酉 & 酉 & 西 & 再 & 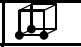 & 酉 & 且 & 17. & 更 & 再 & 西 & II & 通 \\
\hline$\overline{b_{j}^{(\mathrm{L})}}$ & 0 & 0.636 & 0.669 & 1.272 & 1.272 & 0.554 & 1.305 & 1.908 & 0.927 & 0.421 & 1.573 & 1.338 & 2.544 & 1.19 \\
\hline $\overrightarrow{b_{j}}$ & 0 & 376 & 659 & 0.646 & 58 & $\overline{83}$ & 0.768 & 0.813 & 0.92 & 91 & 0.856 & 785 & 0.874 & .8 \\
\hline
\end{tabular}

\section{References}

1. Nagel, W., Ohser, J., Pischang, K.: An integral-geometric approach for the EulerPoincaré characteristic of spatial images. J. Microsc. 198 (2000) 54-62

2. Ohser, J., Nagel, W., Schladitz, K.: The Euler numer of discretized sets - on the choice of adjacency in homogeneous lattices. In Mecke, K.R., Stoyan, D., eds.: Morphology of Condensed Matter, Berlin, Springer-Verlag (2002) 275-298

3. Ohser, J., Nagel, W., Schladitz, K.: The Euler number of discretised sets - surprising results in three dimensions. Image Anal. Stereol. 22 (2003) 11-19

4. Klette, R., Rosenfeld, A.: Digital Geometry. Morgan Kaufmann, San Francisco (2004)

5. Lang, C., Ohser, J., Hilfer, R.: On the analysis of spatial binary images. Journal of Microscopy 203 (2001) 303-313

6. Blasquez, I., Poiraudeau, J.F.: Efficient processing of Minkowski functionals on a 3d binary image using binary decision diagrams. In: Journal of WSCG. Volume 11. Plzen, Czech Republic, WSCG, UNION Agency-Science Press (2003)

7. Schmidt, V., Spodarev, E.: Joint estimators for the specific intrinsic volumes of stationary random sets. Stochastic Process. Appl. 115 (2005) 959-981

8. Windreich, G., Kiryati, N., Lohmann, G.: Surface area estimation in practice. In Nyström, I., di Baja, G.S., Svensson, S., eds.: Discrete Geometry for Computer Imagery. Volume 2886 of LNCS., Berlin, Heidelberg, New York, DGCI, Naples, Italy, Springer (2003) 358-367

9. Lindblad, J.: Surface area estimation of digitized 3D objects using weighted local computations. Image Vision Comp 23 (2005) 111-122

10. Ohser, J., Nagel, W., Schladitz, K.: Miles formulae for Boolean models observed on lattices. In preparation. (2006)

11. Serra, J.: Image Analysis and Mathematical Morphology, volume 1. Academic Press, London (1982)

12. Jernot, J.P., Jouannot-Chesney, P., Lantuéjoul, C.: Local contributions to the Euler-Poincaré characteristic of a set. J. Microsc. 215 (2004) 40-49

13. Lachaud, J.O., Montanvert, A.: Continuous analogs of digital boundaries: A topological approach to iso-surfaces. Graphical Models 62 (2000) 129-164

14. Schneider, R.: Convex Bodies. The Brunn-Minkowski Theory. Cambridge University Press, Cambridge (1993)

15. Weibel, E.R.: Stereological Methods: Theoretical Foundations. Volume 2. Academic Press, London (1980) 